Homology, Homotopy and Applications, vol.12(1), 2010, pp.279-300

\title{
THE CLASSIFYING TOPOS OF A TOPOLOGICAL BICATEGORY
}

\author{
IGOR BAKOVIĆ AND BRANISLAV JURČO
}

(communicated by Ronald Brown)

\begin{abstract}
For any topological bicategory $\mathbb{B}$, the Duskin nerve $N \mathbb{B}$ of $\mathbb{B}$ is a simplicial space. We introduce the classifying topos $\mathcal{B} \mathbb{B}$ of $\mathbb{B}$ as the Deligne topos of sheaves $\operatorname{Sh}(N \mathbb{B})$ on the simplicial space $N \mathbb{B}$. It is shown that the category of geometric morphisms $\operatorname{Hom}(\operatorname{Sh}(X), \mathcal{B B})$ from the topos of sheaves $\operatorname{Sh}(X)$ on a topological space $X$ to the Deligne classifying topos is naturally equivalent to the category of principal $\mathbb{B}$-bundles. As a simple consequence, the geometric realization $|N \mathbb{B}|$ of the nerve $N \mathbb{B}$ of a locally contractible topological bicategory $\mathbb{B}$ is the classifying space of principal $\mathbb{B}$-bundles, giving a variant of the result of Baas, Bökstedt and Kro derived in the context of bicategorical $K$-theory. We also define classifying topoi of a topological bicategory $\mathbb{B}$ using sheaves on other types of nerves of a bicategory given by Lack and Paoli, Simpson and Tamsamani by means of bisimplicial spaces, and we examine their properties.
\end{abstract}

\section{Introduction}

In a recent paper by Baas, Bökstedt and Kro [1], it was shown that the geometric realization $|N \mathbb{B}|$ of the Duskin nerve $N \mathbb{B}[\mathbf{1 1}]$ of a good topological bicategory $\mathbb{B}$ is the classifying space of charted $\mathbb{B}$-bundles. The bicategory is called good if its Duskin nerve $N \mathbb{B}$ is a good simplicial space, i.e., all degeneracy maps are closed cofibrations. Special cases of topological 2-groups and Lie 2-groups were discussed in [2] and in [15], respectively.

The result of $[\mathbf{1}]$ generalizes the well-known fact that the geometric realization $|N \mathbb{C}|$ of the nerve $N \mathbb{C}$ of a locally contractible topological category $\mathbb{C}$ is the classifying space of principal $\mathbb{C}$-bundles (on $\mathrm{CW}$-complexes). This is very nicely described by Moerdijk in $[\mathbf{2 1}]$. The classifying topos $\mathcal{B C}$ of a topological category $\mathbb{C}$ is also described there as the Deligne topos of sheaves $\operatorname{Sh}(N \mathbb{C})$ on the nerve $N \mathbb{C}$, and it is shown that the category of geometric morphisms $\operatorname{Hom}(\operatorname{Sh}(X), \mathcal{B} \mathbb{C})$ from the topos of sheaves $\operatorname{Sh}(X)$ on a topological space $X$ to the Deligne topos is naturally equivalent

The first author was supported by the Croatian Ministry of Science, Education and Sport, Project 098-0982930-2900. He acknowledges support from the European Commission under the MRTN-CT2006-035505 HEPTOOLS.

Received July 16, 2009, revised December 24, 2009; published on April 22, 2010.

2000 Mathematics Subject Classification: 18D05, 18F20, 55U40.

Key words and phrases: bicategory, classifying topos, classifying space, principal bundle.

This article is available at http://intlpress.com/HHA/v12/n1/a14

Copyright (C) 2010, International Press. Permission to copy for private use granted. 
to the category of principal $\mathbb{C}$-bundles. As a simple consequence, it is shown that the geometric realization $|N \mathbb{C}|$ of the nerve $N \mathbb{C}$ of a locally contractible topological category $\mathbb{C}$ is the classifying space of principal $\mathbb{C}$-bundles.

One purpose of this note is to introduce the classifying topos $\mathcal{B B}$ of a topological bicategory $\mathbb{B}$ as the topos of sheaves $\operatorname{Sh}(N \mathbb{B})$ on the Duskin nerve $N \mathbb{B}$ of the bicategory $\mathbb{B}$, which is a simplicial space. The category of geometric morphisms $\operatorname{Hom}(\operatorname{Sh}(X), \mathcal{B B})$ from the topos of sheaves $\operatorname{Sh}(X)$ on a topological space $X$ to the classifying topos is naturally equivalent to the category of (suitably defined) principal $\mathbb{B}$-bundles. As a simple consequence, the geometric realization $|N \mathbb{B}|$ of the nerve $N \mathbb{B}$ of a locally contractible topological bicategory $\mathbb{B}$ is the classifying space of principal $\mathbb{B}$-bundles. Hence, we have a variant of the result of Baas, Bökstedt and Kro.

Another purpose of this note is to define classifying topoi of a topological bicategory $\mathbb{B}$ using sheaves on other types of nerves of the bicategory $\mathbb{B}$, the nerves according to Lack and Paoli $[\mathbf{1 7}]$ (or Simpson [23] and Tamsamani [25]), which can be viewed as bisimplicial spaces. Again, the category of topos morphisms from the topos of sheaves $\operatorname{Sh}(X)$ on a topological space $X$ to the corresponding classifying topos is naturally equivalent to the respective category of (suitably defined) principal $\mathbb{B}$-bundles. As a simple consequence, the geometric realization of any of these nerves of a locally contractible topological bicategory $\mathbb{B}$ is the classifying space of the respective principal $\mathbb{B}$-bundles.

In Section 2, we recall some prerequisites from [21] regarding sheaves on a simplicial space and augmented linear orders over topological spaces. In Section 3, we recall, again from [21], the known facts about classifying spaces and topoi of topological categories (and the corresponding principal bundles). We describe a generalization to the case of bicategories, based on the Duskin nerve, in Section 4. Further preliminaries needed for the subsequent discussion of alternative definitions of classifying spaces and topoi of bicategories are given in Section 5. Finally, in Section 6, we describe a modification of the classifying topos of a topological bicategory (and the corresponding principal bundles) based on alternative definitions of the nerves according to Lack and Paoli, Simpson and Tamsamani.

This article is meant to be the first one in a sequence within a program, initiated by the authors, of classifying topoi of higher order structures in topology. It is a vast generalization of the program initiated by Moerdijk in [21] on the relation between classifying spaces and classifying topoi. Moerdijk's lecture notes arose out of an important question: What does the classifying space of a small category classify? In the article titled by the same question [30], Weiss proved the classifying property of the classifying space for slightly different geometric objects than those of Moerdijk, showing that the answer may not be unique.

Therefore, this article may be seen as an (one possible) answer to the following question: What does the classifying space of a topological bicategory classify? Bicategories are the weakest possible generalization of ordinary categories to the immediate next level of dimension. Like categories, bicategories do have a genuine simplicial set associated with them, their Duskin (geometric) nerve [11]. Based on unpublished work of Roberts on the characterization of the nerve of a strict $n$-category, Street postulated in $[\mathbf{2 4}]$ an equivalence between the category of strict $\omega$-categories and a 
category of certain types of simplicial sets which are called complicial sets. The StreetRoberts conjecture was proved by Verity in [27], and in his subsequent papers, [28] and $[\mathbf{2 9}]$, he gave a characterization of weak $\omega$-categories. Under this characterization, one should be able to capture classifying spaces and topoi of bicategories and other higher-dimensional categories, at least in so far as these concepts have found satisfactory definitions. Following such reasoning, we may define the classifying space of a weak $\omega$-category as the geometric realization of the complicial set, which is its nerve, and the classifying topos of a weak $\omega$-category would be the topos of sheaves on the same complicial set.

It would be interesting to compare this approach to classifying spaces of weak $\omega$-categories with classifying spaces of crossed complexes defined by Brown and Higgins in $[\mathbf{8}]$, since there is a well-known equivalence between strict $\omega$-groupoids and crossed complexes proved in [7] by the same authors. In particular, it would be interesting to see whether the methods we developed would allow one to define a classifying space of a weak $\omega$-category by taking a fundamental crossed complex of its coherent simplicial nerve.

However, this article is not so cosmological in its scope, and its main contribution is to put together some established results on classifying spaces and classifying topoi in a new way, with consequences for the theory of bicategories. Since we are following Moerdijk's approach to classifying spaces and classifying topoi, we will omit all proofs, which can be found in Moerdijk's lecture notes. Many of the definitions and theorems in Sections 2, 3 and 5 are taken almost verbatim from [21].

\section{Acknowledgements}

We would like to thank Ronnie Brown for useful comments on the relevance of this work to general notions of classifying spaces of crossed complexes. We would also like to express our thanks to the referee, whose careful reading resulted in comments and suggestions which have improved the structure and the content of this article. The first author would like to thank the Max Planck Institute for Physics in Munich, where part of this research was done, for its hospitality. He was also supported by the Croatian-German bilateral DAAD program "Homological Algebra in Geometry and Physics" during his stay at the Max Planck Institute for Mathematics in Bonn.

\section{Simplicial spaces and linear orders over topological spaces}

In this section, we recall some prerequisites regarding sheaves on a simplicial space and augmented linear orders over topological spaces. Almost all of the definitions and theorems are taken verbatim from $[\mathbf{2 1}]$, where the proofs of all statements of this section can be found.

\subsection{Topological spaces}

Let us recall that a closed set in a (topological) space $X$ is irreducible if it can not be written as a union of two smaller closed sets. The space $X$ is sober if every irreducible set is the closure $\overline{\{x\}}$ of the one point set $\{x\}$ of a unique $x \in X$. Every Hausdorff space is sober. In this note all spaces will be sober by assumption. 
A space $X$ is locally equiconnected (LEC) if the diagonal map $X \rightarrow X \times X$ is a closed cofibration. For example, CW-complexes are LEC.

A space $X$ is locally contractible if it has a basis of contractible sets. Examples of locally contractible spaces are locally equiconnected spaces and, in particular, CWcomplexes. For a locally contractible space the étale homotopy groups $\pi_{n}\left(\operatorname{Sh}(X), x_{0}\right)$ are naturally isomorphic to the ordinary homotopy groups $\pi_{n}\left(X, x_{0}\right)$ for each $n$.

\subsection{Sheaves as étale spaces}

Throughout this article, we will consider sheaves as sheaves of cross-sections of étale spaces. Recall that a bundle $p: E \rightarrow X$ over $X$ is said to be an étale space over $X$ if for each $e \in E$ there exists an open set $V \subset E$, with $e \in V$, such that $p(V) \subset X$ is open in $X$ and the restriction $\left.p\right|_{V}: V \rightarrow p(V)$ over $V$ is a homeomorphism. There is a well-known equivalence

$$
\operatorname{Etale}(X) \underset{\Lambda}{\stackrel{\Gamma}{\rightleftarrows}} \operatorname{Sh}(X),
$$

where $\Gamma: \operatorname{Etale}(X) \rightarrow \operatorname{Sh}(X)$ is a functor which assigns to each étale space $p: E$ $\rightarrow X$ over $X$ the sheaf of all cross-sections of $E$. The functor $\Lambda: \operatorname{Sh}(X) \rightarrow \operatorname{Etale}(X)$ assigns to each sheaf $S$ the étale space of germes of $S$, where the germ at the point $x \in X$ is an equivalence class germ ${ }_{x} s$ represented by $s \in S(U)$ under the equivalence relation, which relates two elements $s \in S(U)$ and $t \in S(V)$, if there is some open set $W \subset U \cap V$ such that $x \in W$ and $\left.s\right|_{W}=\left.t\right|_{W}$. The stalk of the sheaf $S$ at the point $x \in X$ is the set $S_{x}=\left\{\operatorname{germ}_{x} s: s \in S(U), x \in U\right\}$ of all germs at $x$, which is formally a filtered colimit

$$
S_{x}=\underset{x \in U}{\lim _{x \in U}} S(U)
$$

of the restriction $S^{(x)}: \mathcal{O}_{x}(X)^{\mathrm{op}} \rightarrow$ Set of the sheaf $S$ to the filtered category $\mathcal{O}_{x}(X)^{\mathrm{op}}$ of open neighborhoods of the point $x \in X$. Then $\Lambda S$ is an étale space $p: \coprod_{x \in X} S_{x}$ $\rightarrow X$ whose sheaf of cross sections is canonically isomorphic to $S$. Therefore, we will simultaneously use the terms sheaves and étales spaces in the rest of this article.

\subsection{Topoi}

In the following, a topos will always mean a Grothendieck topos. $\mathrm{Sh}(X)$ will denote topos of sheaves on a (topological) space $X$. A sober space $X$ can be recovered from the topos $\operatorname{Sh}(X)$, which is the faithful image of the space $X$ in the world of topoi.

Further, $\operatorname{Hom}(\operatorname{Sh}(X), \operatorname{Sh}(Y))$ will denote the category of geometric morphisms from $\operatorname{Sh}(X)$ to $\operatorname{Sh}(Y)$. We will also use the same notation $\operatorname{Hom}(\mathcal{F}, \mathcal{E})$ in the more general case of any two topoi $\mathcal{F}$ and $\mathcal{E}$. By definition, a geometric morphism $f \in$ $\operatorname{Hom}(\mathcal{F}, \mathcal{E})$ is a pair of functors $f^{*}: \mathcal{E} \rightarrow \mathcal{F}$ and $f_{*}: \mathcal{F} \rightarrow \mathcal{E}, f^{*}$ being left adjoint to $f_{*}$, and also $f^{*}$ being left exact, i.e., preserving finite limits.

Let us recall that a geometric morphism $f: \mathcal{F} \rightarrow \mathcal{E}$ between locally connected topoi is a weak homotopy equivalence if it induces isomorphisms on étale homotopy (pro)groups $\pi_{0}(\mathcal{F}) \cong \pi_{0}(\mathcal{E})$ and $\pi_{n}(\mathcal{F}, p) \cong \pi_{n}(\mathcal{E}, f q)$, for $n \geqslant 1$ for any basepoint $q \in \mathcal{F}$.

For the collection of homotopy classes of geometric morphism from $\mathcal{F}$ to $\mathcal{E}$ the usual notation $[\mathcal{F}, \mathcal{E}]$ will be used. 


\subsection{The singular functor}

The following construction of a singular functor is taken from [16], where Kelly described it in the context of enriched $\mathcal{V}$-categories for any symmetric monoidal closed category $\mathcal{V}$, which is complete and cocomplete. Let

$$
F: \mathbb{A} \rightarrow \mathbb{E}
$$

be a functor from the small category $\mathbb{A}$. The singular functor of $F$ is the functor

$$
\mathbb{E}(F, 1): \mathbb{E} \rightarrow\left[\mathbb{A}^{\text {op }}, \mathcal{V}\right]
$$

which is obtained as the composite of the Yoneda embedding

$$
\text { Yon: } \mathbb{E} \rightarrow\left[\mathbb{E}^{i \mathrm{op}}, \mathcal{V}\right]
$$

followed by the functor $\left[F^{\mathrm{op}}, \mathcal{V}\right]:\left[\mathbb{E}^{\mathrm{op}}, \mathcal{V}\right] \rightarrow\left[\mathbb{A}^{\mathrm{op}}, \mathcal{V}\right]$ given by restriction along a functor $F$. More explicitly, the singular functor $\mathbb{E}(F, 1)$ sends any object $E$ in $\mathbb{E}$ to the functor

$$
\mathbb{E}(F(-), E): \mathbb{A}^{\text {op }} \rightarrow \mathcal{V},
$$

which takes an object $A$ in $\mathbb{A}$ to the hom-object $\mathbb{E}(F(A), E)$ in $\mathcal{V}$. If the category $\mathbb{E}$ is cocomplete, then the singular functor has a left adjoint

$$
L:\left[\mathbb{A}^{\mathrm{op}}, \mathcal{V}\right] \rightarrow \mathbb{E}
$$

defined for each presheaf $P: \mathbb{A}^{\text {op }} \rightarrow \mathcal{V}$ as the colimit

$$
L(P)=\underline{\lim }\left(\int_{\mathbb{A}} P \stackrel{\pi_{P}}{\longrightarrow} \mathbb{A} \stackrel{F}{\longrightarrow} \mathbb{E}\right),
$$

where $\int_{\mathbb{A}} P$ is the so-called Grothendieck construction $[20]$ on a presheaf $P: \mathbb{A}^{\mathrm{op}} \rightarrow \mathcal{V}$.

\subsection{Grothendieck nerve as a singular functor}

Each ordinal $[n]=\{0<1<\cdots<n\}$ can be seen as a category with the objects $0,1, \ldots, n$ and a unique arrow $i \rightarrow j$ for each $0 \leqslant i \leqslant j \leqslant n$. Also, any monotone map between two ordinals may be seen as a functor. In this way, $\Delta$ becomes a full subcategory of Cat ${ }_{1}$ with a fully faithful inclusion functor

$$
J: \Delta \rightarrow \text { Cat }_{1} .
$$

For any small category $\mathbb{B}$, we see that the composite of the Yoneda embedding Yon $: \mathbb{B} \rightarrow\left[\mathbb{B}^{\text {op }}\right.$, Set $]$, followed by the restriction functor $\left[\mathbb{B}^{\text {op }}\right.$, Set $] \rightarrow\left[\Delta^{\text {op }}\right.$, Set $]$ along $J$, gives a singular functor of $J$. Specifically, the singular functor of $J$ defines the Grothendieck nerve functor

$$
N: \mathrm{Cat}_{1} \rightarrow\left[\Delta^{\mathrm{op}}, \mathrm{Set}\right]
$$

which sends any category $\mathbb{C}$ to the simplicial set $N \mathbb{C}$ which is the nerve of $\mathbb{C}$ whose $n$-simplices are defined by the set

$$
N \mathbb{C}_{n}=[J([n]), \mathbb{C}],
$$

where the right side denotes the set of functors from an ordinal $[n]$ to the category $\mathbb{C}$. The nerve functor is fully faithful, which means that the simplicial skeletal category $\Delta$ is an adequate subcategory of the category Cat $_{1}$ in the sense of Isbell $[\mathbf{1 3}, \mathbf{1 4}]$. We also say that the corresponding embedding is dense, in the sense of Kelly [16]. 


\subsection{Simplicial spaces}

Let $\Delta$ be the simplicial model category having as objects the nonempty finite sets (ordinals) $[n]=\{0,1, \ldots, n\}$, for $n \geqslant 0$, and as arrows the order-preserving functions $\alpha:[n] \rightarrow[m]$. A simplicial space (set) is a contravariant functor from $\Delta$ into the category of spaces (sets). Its value at $[n]$ is denoted $Y_{n}$, and its action on the arrow $\alpha:[n] \rightarrow[m]$ is denoted as $Y(\alpha): Y_{m} \rightarrow Y_{n}$. A simplicial space $Y$ is called locally contractible if each $Y_{n}$ has a basis of contractible sets.

For a simplicial space $Y$, the geometric realization $|Y|$ will always mean the thickened (fat) geometric realization. This is defined as a topological space obtained from the disjoint sum $\sum_{n \geqslant 0} X_{n} \times \Delta^{n}$ by the the equivalence relations

$$
\left(\alpha^{*}(x), t\right) \sim(x, \alpha(t))
$$

for all injective (order-preserving) arrows $\alpha:[n] \rightarrow[m] \in \Delta$, any $x \in X_{m}$ and any $t \in \Delta^{n}$, where $\Delta^{n}$ is the standard topological $n$-simplex. If all degeneracies are closed cofibrations, i.e., the simplicial space is a good simplicial space, then this geometric realization is homotopy equivalent to the geometric realization of the underlying simplicial set of $Y$, which is defined as above but allowing for all arrows in $\Delta$. In particular, $Y$ is good if all spaces $Y_{n}$ are locally equiconnected [1]. Geometric realization of a locally contractible simplicial space is a locally contractible space.

Definition 2.1. A sheaf $S$ on a simplicial space $Y$ is defined to be a system of sheaves $S^{n}$ on $Y_{n}$, for $n \geqslant 0$, together with sheaf maps $S(\alpha): Y(\alpha)^{*} S^{n} \rightarrow S^{m}$ for each $\alpha:[n] \rightarrow[m]$. These maps are required to satisfy the following functoriality conditions:

(i) (normalization). $S\left(\operatorname{id}_{[n]}\right)=\operatorname{id}_{S_{n}}$, and

(ii) for any $\alpha:[n] \rightarrow[m], \beta:[m] \rightarrow[k]$ the following diagram:

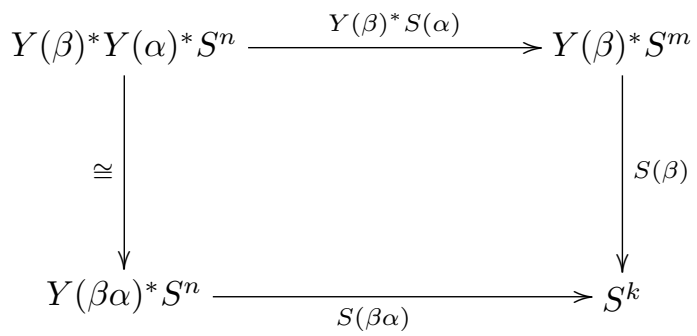

is commutative. A morphism $f: S \rightarrow T$ of sheaves on $Y$ consists of the maps $f_{n}: S^{n} \rightarrow$ $T^{n}$ of sheaves on $Y_{n}$ for each $n \geqslant 0$, which are compatible with the structure maps $S(\alpha)$ and $T(\alpha)$. This defines the category $\operatorname{Sh}(Y)$ of sheaves on the simplicial space $Y$.

Proposition 2.2. The category $\mathrm{Sh}(Y)$ of sheaves on a simplicial space is a topos.

Theorem 2.3. For any simplicial space $Y$, the topoi $\operatorname{Sh}(Y)$ and $\operatorname{Sh}(|Y|)$ have the same weak homotopy type.

Definition 2.4. A linear order over a topological space $X$ is a sheaf $p: L \rightarrow X$ on $X$ together with a subsheaf $O \subseteq L \times_{X} L$ such that for each point $x \in X$ the stalk 
$L_{x}$ is nonempty and is linearly ordered by the relation

$$
y \leqslant z \quad \text { if and only if } \quad(y, z) \in O_{x},
$$

for $y, z \in L_{x}$. A mapping $L \rightarrow L^{\prime}$ between two linear orders over $X$ is a mapping of sheaves restricting for each $x \in X$ to an order-preserving map of stalks $L_{x} \rightarrow L_{x}^{\prime}$. This defines a category of linear orders on $X$.

Example 2.5. An open ordered covering $\mathcal{U}=\left\{U_{i}\right\}_{i \in I}$ of a topological space $X$ is a covering indexed over a partially ordered set $I$, which restricts to a total ordering on every finite subset $\left\{i_{0}, \ldots, i_{n}\right\}$ of $I$ whenever the finite intersection $U_{i_{0}, \ldots, i_{n}}=$ $U_{i_{0}} \cap \cdots \cap U_{i_{n}}$ is nonempty. When a sheaf $p: L \rightarrow X$ is given by the projection $p: \coprod_{i \in I} U_{i} \rightarrow X$ from the disjoint union of open sets in the open ordered covering $\mathcal{U}$, the subsheaf $p^{[2]}: L \times_{X} L \rightarrow X$ is given by the induced projection $p^{[2]}: \coprod_{i, j \in I} U_{i j}$ $\rightarrow X$ from the family $\left\{U_{i j}\right\}_{i, j \in I}$ of double intersections of open sets $\mathcal{U}$. The family of inclusions $i_{i j}: U_{i j} \hookrightarrow \coprod_{i, j \in I} U_{i j}$, for each $U_{i j} \neq \emptyset$ such that $i<j$, defines a subsheaf $O=\coprod_{i<j} U_{i j}$ of $L=\coprod_{i, j \in I} U_{i j}$ whose stalks $O_{x}$ are linearly ordered for any $x \in X$. Therefore, open ordered coverings used by Baas, Bökstedt and Kro in [1] are examples of linear orders over $X$.

Remark 2.6. A linear order $L$ over $X$ defines an obvious topological category with $L$ as the space of objects and the order subsheaf $O \subseteq L \times_{X} L$ as the space of arrows. Hence, we can speak of a nerve $N L$ of the linear order $L$. This nerve is obviously a simplicial sheaf on $X$ (a simplicial space with étale maps into $X$ ).

Recall that any open covering of a topological space $X$ can be assembled into a simplicial sheaf over $X$ with distinguished properties. Therefore, by the construction in Example 2.5 and following Remark 2.6, we may regard linear orders as generalizations of coverings of topological spaces.

Definition 2.7. For any space $X$ and any simplicial space $Y$, write $\operatorname{Lin}(X, Y)$ for the category of linear orders $(L$, aug) over $X$ equipped with a simplicial map (augmentation) aug: $N L \rightarrow Y$ from the nerve of $L$ to $Y$. A morphism $(L$, aug $) \rightarrow\left(L^{\prime}\right.$, aug' $)$ in $\operatorname{Lin}(X, Y)$ are maps of linear orders $L \rightarrow L^{\prime}$ such that the induced map $N L \rightarrow N L^{\prime}$ on the nerves respects the augmentations.

If we regard linear orders as generalizations of coverings of topological spaces, then augmentations of linear orders may be seen as cocycles on such coverings.

Example 2.8. Let $N \mathbb{C}$ be the nerve of a topological category $\mathbb{C}$. An augmentation aug: $N L \rightarrow N \mathbb{C}$ of a linear order $L$ defined by an open ordered covering $\mathcal{U}=\left\{U_{i}\right\}_{i \in I}$ of a topological space $X$, as in Example 2.5, is a Čech cocycle on the covering $\mathcal{U}$ with values in the category $\mathbb{C}$.

Definition 2.9. We call two objects $E_{0}, E_{1} \in \operatorname{Lin}(X, Y)$ concordant if there exists an $E \in \operatorname{Lin}(X \times[0,1], Y)$ such that we have $E_{0} \cong i_{0}^{*}(E)$ and $E_{0} \cong i_{1}^{*}(E)$ under the obvious inclusions $i_{0}, i_{1}: X \hookrightarrow X \times[0,1]$. $\operatorname{Lin}_{c}(X, Y)$ will denote the collection of concordance classes of objects from $\operatorname{Lin}(X, Y)$. 
Theorem 2.10. Let $Y$ be a simplicial space. For any space $X$ there is a natural equivalence of categories

$$
\operatorname{Hom}(\operatorname{Sh}(X), \operatorname{Sh}(Y)) \simeq \operatorname{Lin}(X, Y) .
$$

On homotopy classes of topos morphisms we have the natural bijection

$$
[\operatorname{Sh}(X), \operatorname{Sh}(Y)] \cong \operatorname{Lin}_{c}(X, Y) .
$$

Corollary 2.11. Let $X$ be a $\mathrm{CW}$-complex and $Y$ be a locally contractible simplicial space. There is a natural bijection between homotopy classes of maps $[X,|Y|]$ and concordance classes $\operatorname{Lin}_{c}(X, Y)$.

Remark 2.12. If, in addition, the simplicial space $Y$ is a good one, then the above is also true if we use the geometric realization of the underlying simplicial set of $Y$ instead of its thickened geometric realization. In particular, it does not matter which geometric realization we use if each of $Y_{n}$ is LEC or a CW-complex.

\section{Classifying spaces and classifying topoi of topological cat- egories}

In this section we specify the known results described in Section 1 to the case when the simplicial space $Y$ is the nerve of a topological category $\mathbb{C}$. The reader who is interested in more details is referred to [21], which we again follow almost verbatim.

Definition 3.1. Let $\mathbb{C}$ be a topological category. The classifying topos $\mathcal{B} \mathbb{C}$ of a topological category is defined as the topos $\operatorname{Sh}(N \mathbb{C})$.

Definition 3.2. The classifying space $B \mathbb{C}$ of a topological category $\mathbb{C}$ is the geometric realization $|N \mathbb{C}|$ of its nerve $N \mathbb{C}$.

With these definitions we have the following corollary of Theorem 2.3.

Corollary 3.3. For any topological category $\mathbb{C}$, the topos of sheaves $\operatorname{Sh}(B \mathbb{C})$ on the classifying space $B \mathbb{C}$ has the same weak homotopy type as the classifying topos $\mathcal{B} \mathbb{C}$.

Definition 3.4. For any topological category $\mathbb{C}$, write $\operatorname{Lin}(X, \mathbb{C})$ for the category of linear orders over $X$ equipped with an augmentation $N L \rightarrow N \mathbb{C}$. An object $E$ of this category will be called a principal $\mathbb{C}$-bundle. We call two principal $\mathbb{C}$-bundles $E_{0}$ and $E_{1}$ on $X$ concordant if there exists a principal $\mathbb{C}$-bundle on $X \times[0,1]$ such that we have isomorphisms $E_{0} \cong i_{0}^{*}(E)$ and $E_{0} \cong i_{1}^{*}(E)$ under the obvious inclusions $i_{0}, i_{1}: X \hookrightarrow X \times[0,1]$.

Remark 3.5. The nerve construction leads to a bijection between principal $\mathbb{C}$-bundles and linear orders $L$ equipped with a continuous functor $L \rightarrow \mathbb{C}$.

The fact that the classifying topos $\mathcal{B} \mathbb{C}$ classifies principal $\mathbb{C}$-bundles now follows immediately from Theorem 2.10 . 
Theorem 3.6. For a topological category $\mathbb{C}$ and a topological space $X$, there is a natural equivalence of categories

$$
\operatorname{Hom}(\operatorname{Sh}(X), \mathcal{B} \mathbb{C}) \simeq \operatorname{Lin}(X, \mathbb{C}) .
$$

On homotopy classes of topos morphisms, we have the natural bijection

$$
[\operatorname{Sh}(X), \mathcal{B} \mathbb{C}] \cong \operatorname{Lin}_{c}(X, \mathbb{C}) .
$$

Similarly, the fact that the classification space $B \mathbb{C}$ classifies principal $\mathbb{C}$-bundles now follows from Corollary 2.11.

Definition 3.7. We say that a topological category $\mathbb{C}$ is locally contractible if its space of objects $C_{0}$ and its space of arrows $C_{1}$ are locally contractible. A topological category $\mathbb{C}$ is a good topological category, if its nerve $N \mathbb{C}$ is a good simplicial space.

Corollary 3.8. For a locally contractible category $\mathbb{C}$ and a $\mathrm{CW}$-complex $X$, there is a natural bijection

$$
[X, B \mathbb{C}] \cong \operatorname{Lin}_{c}(X, \mathbb{C})
$$

Remark 3.9. If, in addition, the topological category $\mathbb{C}$ is a good one, then the above is also true if we use the geometric realization of the underlying simplicial set instead of the thickened geometric realization of the nerve. In particular, it does not matter which geometric realization we use if all $N \mathbb{C}_{n}$ are LEC.

\section{Classifying spaces and classifying topoi of topological bicat- egories I}

In this section we specify the known results described in Section 1 to the case when the simplicial space $Y$ is the nerve of a topological bicategory $\mathbb{B}$.

\subsection{Duskin nerve as a singular functor}

The Duskin nerve [11] can also be obtained as a singular functor when we take $\mathcal{V}=$ Set. Every category (in particular the category defined above by the ordinal $[n]$ ) can be seen as a locally discrete bicategory (the only 2-cells are identities) which gives a fully faithful inclusion

$$
H: \Delta \rightarrow \text { Bicat }_{1}
$$

where Bicat ${ }_{1}$ denotes the category of bicategories and normal lax functors or normal morphisms of bicategories defined by Bénabou in [6]. The singular functor of the inclusion $H$ is the Duskin nerve functor

$$
N: \text { Bicat }_{1} \rightarrow\left[\Delta^{\mathrm{op}}, \text { Set }\right],
$$

which is fully faithful and sends a (small) bicategory $\mathbb{B}$ to its nerve $N \mathbb{B}$ which is a simplicial set whose $n$-simplices are defined by the set

$$
N \mathbb{B}_{n}=[H([n]), \mathbb{B}] .
$$

The right side is a set of normal lax functors from an ordinal $[n]$ to the bicategory $\mathbb{B}$. 
Definition 4.1. For an ordinal $[n]$ and a bicategory $\mathbb{B}$, a normal lax functor $(B, f, \beta):[n] \rightarrow \mathbb{B}$ consists of the following data in $\mathbb{B}$ :

(i) an object $B_{i}$ for each $i \in[n]$,

(ii) a morphism $f_{i j}: B_{i} \rightarrow B_{j}$ for each $i, j \in[n]$ with $i \leqslant j$,

(iii) a 2-cell $\beta_{i j k}: f_{i j} \circ f_{j k} \Rightarrow f_{i k}$ for each $i, j, k \in[n]$ with $i \leqslant j \leqslant k$

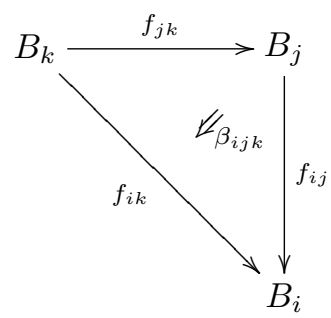

such that the following conditions are satisfied:

- (normalization). For any $i \in[n]$ we have $f_{i i}=i_{B_{i}}: B_{i} \rightarrow B_{i}$, and for any $i, j \in[n]$, such that $i \leqslant j$, the corresponding 2-cells $\beta_{i i j}: f_{i i} \circ f_{i j} \Rightarrow f_{i j}$ and $\beta_{i j j}: f_{i j} \circ f_{j j} \Rightarrow f_{i j}$ are given by the two 2 -simplices
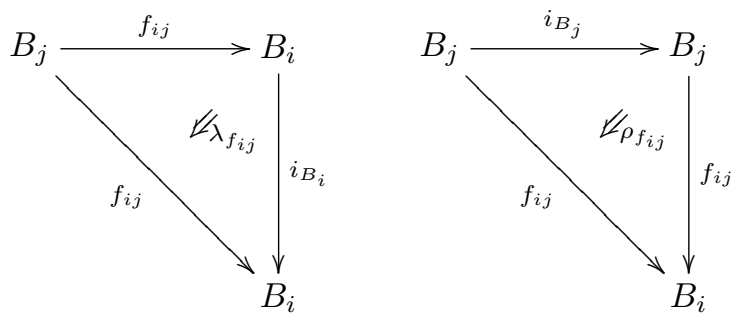

where $\rho_{f_{i j}}: f_{i j} \circ i_{p_{j}} \Rightarrow f_{i j}$ and $\lambda_{f_{i j}}: i_{p_{i}} \circ f_{i j} \Rightarrow f_{i j}$ are the components of the right and left identity natural isomorphisms in $\mathbb{B}$.

- (coherence condition). For each $i, j, k, l \in[n]$ such that $i \leqslant j \leqslant k \leqslant l$ the following tetrahedron:

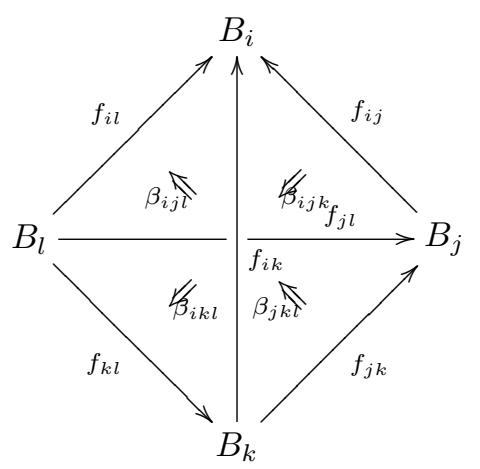

is commutative. This means that we have the identity of 2-cells in the bicategory $\mathbb{B}$ :

$$
\beta_{i k l}\left(\beta_{i j k} \circ f_{k l}\right)=\beta_{i j l}\left(f_{i j} \circ \beta_{j k l}\right) \alpha_{i j k l} .
$$


Remark 4.2. Simplicial sets that are isomorphic to a nerve of a bicategory have been characterized in [11] and [12]. Simplicial sets that are isomorphic to a nerve of a bicategory form a full subcategory of the category of simplicial sets. This category is equivalent to the category Bicat ${ }_{1}$ of bicategories with lax normal functors. Let us recall that a lax functor $(F, \phi)$ is normal if $F\left(\operatorname{id}_{x}\right)=\operatorname{id}_{F x}$ and $\phi_{x}: \mathrm{id}_{F x} \Longrightarrow F\left(\operatorname{id}_{x}\right)$ is the identity 2-cell, and that oplax means that all the structure maps go in the opposite direction. This equivalence also holds in the topological setting.

Definition 4.3. Let $\mathbb{B}$ be a topological bicategory. The classifying topos $\mathcal{B} \mathbb{B}$ of the topological bicategory $\mathbb{B}$ is defined as the topos $\operatorname{Sh}(N \mathbb{B})$.

Definition 4.4. The classifying space $B \mathbb{B}$ of a topological bicategory $\mathbb{B}$ is the geometric realization $|N \mathbb{B}|$ of its nerve $N \mathbb{B}$.

With these definitions we have the following corollary of Theorem 2.3.

Corollary 4.5. For any topological bicategory $\mathbb{B}$, the topos of sheaves $\operatorname{Sh}(B \mathbb{B})$ on the classifying space $B \mathbb{B}$ has the same weak homotopy type as the classifying topos $\mathcal{B} \mathbb{B}$.

Definition 4.6. For a topological bicategory $\mathbb{B}$ write $\operatorname{Lin}(X, \mathbb{B})$ for the category of linear orders over $X$ equipped with an augmentation aug: $N L \rightarrow N \mathbb{B}$. An object $E$ of this category will be called a Duskin principal $\mathbb{B}$-bundle. We call two Duskin principal $\mathbb{B}$-bundles $E_{0}$ and $E_{1}$ on $X$ concordant, if there exists a Duskin principal $\mathbb{B}$-bundle on $X \times[0,1]$ such that we have the equivalences $E_{0} \simeq i_{0}^{*}(E)$ and $E_{0} \simeq i_{1}^{*}(E)$ under the obvious inclusions $i_{0}, i_{1}: X \hookrightarrow X \times[0,1]$.

Remark 4.7. We can consider a linear order $L$ as a locally trivial bicategory (with only trivial 2-morphisms). In this case the Duskin nerve of $L$ coincides with the ordinary nerve of $L$ which justifies the same notation $N L$ for both nerves.

Remark 4.8. By the above remark, an augmentation $N L \rightarrow N \mathbb{B}$ is the same, by the nerve construction, as a continuous normal lax functor $L \rightarrow \mathbb{B}$.

Similarly to Theorem 3.6, we have from Theorem 2.10 the following "classifying" property of the classifying 1-topos $\mathcal{B} \mathbb{B}$.

Theorem 4.9. For a topological bicategory $\mathbb{B}$ and a topological space $X$, there is a natural equivalence of categories

$$
\operatorname{Hom}(\operatorname{Sh}(X), \mathcal{B B}) \simeq \operatorname{Lin}(X, \mathbb{B}) .
$$

On homotopy classes of topos morphisms we have the natural bijection

$$
[\operatorname{Sh}(X), \mathcal{B B}] \cong \operatorname{Lin}_{c}(X, \mathbb{B}) .
$$

Definition 4.10. We say that a topological bicategory $\mathbb{B}$ is locally contractible $\mathbb{B}$ if its space of objects $B_{0}$, its space of 1 -arrows $B_{1}$ and its space of 2-arrows $B_{2}$ are locally contractible. A topological bicategory $\mathbb{B}$ is a good topological bicategory, if its nerve $N \mathbb{B}$ is a good simplicial space.

The "classification" property of the classifying space $B \mathbb{B}$ now follows as a corollary from Corollary 2.11. 
Corollary 4.11. For a locally contractible bicategory $\mathbb{B}$ and a $\mathrm{CW}$-complex $X$, there is a natural bijection

$$
[X, B \mathbb{B}] \cong \operatorname{Lin}_{c}(X, \mathbb{B}) .
$$

Remark 4.12. If, in addition, the topological bicategory $\mathbb{B}$ is a good one, then the above is also true if we use the geometric realization of the underlying simplicial set instead of the thickened geometric realization of the nerve. In particular, it does not matter which geometric realization we use if all $N \mathbb{B}_{n}$ are LEC. The case of a good topological bicategory, as well as the sufficient conditions for a bicategory being a good one, are discussed in $[\mathbf{1}]$. Those conditions actually guarantee that all $N \mathbb{B}_{n}$ are LEC. Thus, our corollary above gives a slight generalization of the result of Baas, Bökstedt and Kro.

\section{Principal bundles under a category}

Before introducing an alternative notion of a classifying topos of a bicategory in the next section, we will introduce some additional background material. Everything up to and including Remark 5.18 is taken almost verbatim from [21], where the interested reader can find the missing proofs (as well as more details). Definition 5.20, Theorem 5.21 and Corollary 5.23 might be new. To make our discussion more complete, we start with the definitions of the classifying topoi in the cases of a small and $s$-étale category. We also recall the definition of a principal $\mathbb{C}$-bundles in these cases.

Proposition 5.1. The category of all presheaves on a small category $\mathbb{C}$ is a topos.

Definition 5.2. The topos $\mathcal{B} \mathbb{C}$ of presheaves on a small category $\mathbb{C}$ is called the classifying topos of $\mathbb{C}$.

Remark 5.3. At this point, the reader may wonder how the above Definition 5.2 is related to the definition of the classifying topos of a topological category given in Definition 3.1. We will address this question later in 5.11, after we introduce further relevant material.

Definition 5.4. For a small category $\mathbb{C}$ and a space $X$, a $\mathbb{C}$-bundle over $X$ is a covariant functor $E: \mathbb{C} \rightarrow \operatorname{Sh}(X)$. Such a $\mathbb{C}$-bundle is called a principal (flat, filtering) if for each point $x \in X$ the following conditions - nonemptiness, transitivity and freeness - are satisfied for the stalks $E(c)_{x}$ for objects $c \in \mathbb{C}$ :

(i) There is at least one object $c$ in $\mathbb{C}$ for which the stalk $E(c)_{x}$ is nonempty.

(ii) For any two points $y \in E(c)_{x}$ and $z \in E(d)_{x}$, there are arrows $\alpha: b \rightarrow c$ and $\beta: b \rightarrow d$ from some object $b$ of $\mathbb{C}$, and an object $w \in E(b)_{x}$ such that $\alpha w=y$ and $\beta w=z$.

(iii) For any two parallel arrows $\alpha, \beta: c \rightarrow d$ and any $y \in E(c)_{x}$ for which $\alpha y=\beta y$, there is an arrow $\gamma: b \rightarrow c$ and a point $z \in E(b)_{x}$ such that $\alpha \gamma=\beta \gamma$ and $\gamma z=y$.

A map between two principal $\mathbb{C}$-bundles is a natural transformation between the corresponding functors. The category of principal $\mathbb{C}$-bundles will be denoted as $\operatorname{Prin}(X, \mathbb{C})$. 
Examples 5.5. The following well-known notions are examples of principal $\mathbb{C}$-bundles:

(i) (principal group bundles). Any group $G$ can be seen as a groupoid (and therefore a category) with only one object. In this way, the above definition of a principal $\mathbb{C}$-bundle becomes the usual one, where a principal left $G$-bundle over $X$ is an étale space $p: P \rightarrow X$ with a fibre-preserving left action $a: G \times P \rightarrow P$ of $G$ on $P$ for which the induced map $\left(a, p r_{2}\right): G \times P \rightarrow P \times P$ is a homeomorphism.

(ii) (principal monoid bundles). Any monoid $M$ can be seen as a category with only one object. If every morphism in a such category is a monomorphism, then the monoid $M$ is said to have left cancellation if $m k=m l$ implies $k=l$ for any $k, l, m \in M$. Segal used such a monoid $M$ in order to introduce a right principal monoid bundle in [22] as an étale space $p: P \rightarrow X$ over $X$ with a fibre-preserving right action of $M$ on $P$, such that each fibre $P_{x}$ is a principal $M$-set. A right principal $M$-set $S$ is a set with a right action of $M$, which is free in the sense that $s m_{1}=s m_{2}$ for any $m_{1}, m_{2} \in M$ and $s \in S$, and is transitive in the sense that for any $s_{1}, s_{2} \in M$ there exist $m_{1}, m_{2} \in M$ and $s \in S$ such that $s_{1}=s m_{1}$ and $s_{2}=s m_{2}$. Although Segal used the right action of a monoid with left cancellation, it is obvious that when $\mathbb{C}$ is a monoid $M$ with right cancellation, the above definition of a left principal $\mathbb{C}$-bundle becomes a left principal monoid bundle.

(iii) (principal poset bundles). Any partially ordered set $P$ may be seen as a category with exactly one morphism $i \rightarrow j$ if and only if $i \leqslant j$. A principal $P$-bundle over a topological space $X$ is a covering $\mathcal{U}=\left\{U_{i}\right\}_{i \in P}$ of $X$ such that when $i \leqslant j$ then $U_{i} \subseteq U_{j}$ and which is locally directed in the sense that any $U_{i j}=U_{i} \cap U_{j}$ is covered by the family $\mathcal{U}_{i j}=\left\{U_{k}: k \leqslant i \wedge k \leqslant j\right\}$.

(iv) (principal simplicial sets). Any linear order over a topological space defines a topological category and therefore a simplicial space via its nerve as in Remark 2.6. One can see that a simplicial set $S: \Delta^{\mathrm{op}} \rightarrow$ Set is a principal $\Delta^{\mathrm{op}}$-bundle if and only if is the nerve of a (uniquely determined) nonempty linear order.

Definition 5.6. A $\mathbb{C}$-sheaf is an étale space $p: S \rightarrow C_{0}$ equipped with a continuous right $\mathbb{C}$ action

$$
\alpha: S \times_{C_{0}} C_{1} \rightarrow S
$$

which we denote by $\alpha(x, f)=x \cdot f$. This action is defined for all pairs $(x, f)$ for which $p(x)=t(f)$, and it satisfies the following axioms:

$$
p(x \cdot f)=s(f), \quad(x \cdot f) \cdot g=x \cdot(f g), \quad x \cdot \operatorname{id}_{p(x)}=x .
$$

A map between $\mathbb{C}$-sheaves or a $\mathbb{C}$-equivariant map is a map of étale spaces over $C_{0}$, which is compatible with the $\mathbb{C}$-action.

Proposition 5.7. The category of $\mathbb{C}$-sheaves is a topos.

Definition 5.8. The topos $\mathcal{B C}$ of $\mathbb{C}$-sheaves is called the classifying topos of the $s$-étale topological category $\mathbb{C}$.

Examples 5.9. We now provide some examples of $\mathbb{C}$-sheaves to illustrate their significance: 
(i) Any small category $\mathbb{C}$ can be seen as a topological category with the discrete topology. Then a $\mathbb{C}$-sheaf is the same thing as a presheaf on $\mathbb{C}$ which justifies the same notation $\mathcal{B} \mathbb{C}$, as in Definition 5.2 , for the classifying topos of a small category $\mathbb{C}$.

(ii) Any topological space $X$ may be seen as a discrete topological category $\mathbb{X}$ (the one for which all morphisms are identities). Then an $\mathbb{X}$-sheaf is just a sheaf on $X$ and the topos $\mathcal{B} \mathbb{X}$ is the topos $\operatorname{Sh}(X)$ of sheaves on $X$.

(iii) Let $\mathcal{G}$ be an action groupoid coming from the right action of a topological group $G$ on a topological space $X$. The groupoid $\mathcal{G}$ has $X$ as a space of objects and $X \times G$ as a space of morphisms, where morphisms are of the form $(x, g): x \cdot g$ $\rightarrow x$. Then a $\mathcal{G}$-sheaf $p: S \rightarrow X$ is a sheaf which is $G$-equivariant. Therefore, $\mathcal{B G}$ is the category of $G$-equivariant sheaves.

In the case of an $s$-étale topological category, i.e., a topological category with the source map $s: C_{1} \rightarrow C_{0}$ being an étale map, we have the following definition.

Definition 5.10. Let $\mathbb{C}$ be an $s$-étale topological category. A $\mathbb{C}$-bundle over a space $X$ is an étale map (sheaf) $p: E \rightarrow X$ with a continuous fibrewise left action given by the maps

$$
\pi: E \rightarrow B_{0}, \quad \text { and } \quad a: B_{1} \times_{B_{0}} E \rightarrow E .
$$

Such a $\mathbb{C}$-bundle is called principal if the three conditions of nonemptiness, transitivity and freeness hold for each $x \in X$ :

(i) The stalk $E_{x}$ is nonempty.

(ii) For any two points $y \in E_{x}$ and $z \in E_{x}$, there are a $w \in E_{x}$ and arrows $\alpha: \pi(w)$ $\rightarrow \pi(y)$ and $\beta: \pi(w) \rightarrow \pi(z)$ such that $\alpha w=y$ and $\beta w=z$.

(iii) For any point $y \in E_{x}$ and any pair of arrows $\alpha, \beta$ in $\mathbb{B}$ with $s(\alpha)=\pi(y)=s(\beta)$ and $\alpha y=\beta y$, there is a point $w \in E_{x}$ and an arrow $\gamma: \pi(w) \rightarrow \pi(y)$ in $\mathbb{C}$ such that $\gamma w=y$ in $E_{x}$ and $\alpha \gamma=\beta \gamma$ and $\gamma z=y$ in $\mathbb{B}$.

A map between two principal $\mathbb{C}$-bundles is a sheaf map preserving the $\mathbb{C}$-action. The resulting category of principal $\mathbb{C}$-bundles will again be denoted as $\operatorname{Prin}(X, \mathbb{C})$.

Remark 5.11. A small category can be viewed as an $s$-étale topological category with the discrete topology. In this case the respective definitions of principal bundles and of classifying topoi are of course equivalent. A topological category is locally connected if the spaces of objects and arrows are locally connected. For a locally connected $s$-étale topological category, the classifying topos introduced in this section and the one defined as the topos of sheaves on the nerve are weak homotopy equivalent.

In both cases (small and s-étale topological) we have the same notion of concordance of principal $\mathbb{C}$-bundles as in topological case (see 2.9).

For either a small or an $s$-étale topological category, we have: 
Theorem 5.12. There is a natural equivalence of categories

$$
\operatorname{Hom}(\operatorname{Sh}(X), \mathcal{B} \mathbb{C}) \simeq \operatorname{Prin}(X, \mathbb{C}) .
$$

On homotopy classes of topos morphisms we have the natural bijection

$$
[\operatorname{Sh}(X), \mathcal{B} \mathbb{C}] \cong \operatorname{Prin}_{c}(X, \mathbb{C}) .
$$

For a $\mathrm{CW}$-complex $X$ and any small category or any locally contractible s-étale category $\mathbb{C}$, there is a natural bijection

$$
[X, B \mathbb{C}] \cong \operatorname{Prin}_{c}(X, \mathbb{C}),
$$

where in the s-étale case the fat geometric realization is taken in order to construct the classifying space.

Proposition 5.13. For either a small category or a locally connected s-étale category, there is a natural weak homotopy equivalence

$$
\operatorname{Sh}(B \mathbb{C}) \rightarrow \mathcal{B C} .
$$

Remark 5.14. Definition 2.1 of the topos $\operatorname{Sh}(Y)$ of sheaves on the simplicial space $Y$ generalizes to the case when the opposite simplicial model category $\Delta^{\mathrm{op}}$ is replaced by an arbitrary small category $\mathbb{K}$. Then, instead of a simplicial space, we have a diagram of spaces indexed by $\mathbb{K}$, i.e., a covariant functor $Y$ from $\mathbb{K}$ into the category Top topological spaces. With an evident modification of Definition 2.6, we obtain the topos of sheaves on the diagram of spaces $Y$.

Remark 5.15. From a diagram of spaces indexed by a small category $\mathbb{K}$, we can construct a category $Y_{\mathbb{K}}$. The object is a pair $(k, y), k \in \mathbb{K}, y \in Y_{k}$ and the arrow $(k, y) \rightarrow(l, z)$ is an arrow in $\mathbb{K} \alpha: k \rightarrow l$ such that $Y(\alpha)(y)=z$. This is just the Grothendieck construction. The category $Y_{\mathbb{K}}$ can be equipped with an $s$-étale topology. Further, a diagram of spaces $Y$ is called locally contractible if each $Y_{k}$ is locally contractible. For a locally contractible $Y$, the Grothendieck construction gives a locally contractible $s$-étale topological category $Y_{\mathbb{K}}$.

Proposition 5.16. Let $\operatorname{Sh}(Y)$ be the category of sheaves on a diagram of spaces $Y$ indexed by a small category $\mathbb{K}$. Then there is a natural equivalence of topoi

$$
\operatorname{Sh}(Y) \simeq \mathcal{B}\left(Y_{\mathbb{K}}\right) .
$$

Hence, for any topological space $X$, there is a natural equivalence

$$
\operatorname{Hom}(\operatorname{Sh}(X), \operatorname{Sh}(Y)) \simeq \operatorname{Prin}\left(X, Y_{\mathbb{K}}\right) .
$$

A principal $Y_{\mathbb{K}}$-bundle can also be characterized as a principal $\mathbb{K}$-bundle equipped with an augmentation. Let us recall that a principal $\mathbb{K}$-bundle over $X$ consists of a system of sheaves $E^{k}$ for each object $k$ of $\mathbb{K}$ on $X$ and sheaf maps $E(\alpha): E^{k} \rightarrow E^{l}$ for each arrow $\alpha: k \rightarrow l$. An augmentation on of $E$ over $Y$ is a system of maps $\operatorname{aug}^{k}: E^{k} \rightarrow Y_{k}$ such that for any arrow $\alpha: k \rightarrow l$

$$
Y(\alpha) \operatorname{aug}^{k}=\operatorname{aug}^{l} E(\alpha) .
$$

Together with the morphisms of principal bundles that respect augmentations, we 
have the category

$$
\operatorname{Aug} \operatorname{Prin}(X, \mathbb{K}, Y)
$$

of principal $\mathbb{K}$-bundles with an augmentation to $Y$.

Proposition 5.17. For $X$ and $Y$ as above, we have a natural equivalence of categories

$$
\operatorname{Hom}(\operatorname{Sh}(X), \operatorname{Sh}(Y)) \simeq \operatorname{Prin}\left(X, Y_{\mathbb{K}}\right) \simeq \operatorname{AugPrin}(X, \mathbb{K}, Y)
$$

Remark 5.18. The case $\mathbb{K}=\Delta^{\mathrm{op}}$ gives Theorem 2.10 as a corollary. For this, the following equivalence

$$
\operatorname{Prin}\left(X, \Delta^{\mathrm{op}}\right) \simeq \operatorname{Lin}(X)
$$

has to be used. A principal $\Delta^{\text {op }}$-bundle $E$ over $X$ is a simplicial sheaf such that each

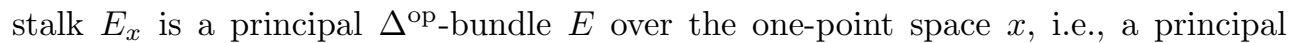
simplicial set. Finally, a simplicial set is principal only if it is a nerve of a (uniquely determined) nonempty linear order.

Next, let us consider the case $\mathbb{K}=\Delta^{\mathrm{op}} \times \Delta^{\mathrm{op}}$; i.e., in this case a diagram of spaces $Y$ labeled by $\Delta^{\mathrm{op}} \times \Delta^{\mathrm{op}}$ is just a bisimplicial space. Concerning principal $\Delta^{\mathrm{op}} \times \Delta^{\mathrm{op}}$ bundles over $X$, we have the following result which follows from [20, Chap. VII, exercise 14].

Proposition 5.19. There are natural equivalences of categories

$$
\operatorname{Prin}\left(X, \Delta^{\mathrm{op}} \times \Delta^{\mathrm{op}}\right) \simeq \operatorname{Prin}\left(X, \Delta^{\mathrm{op}}\right) \times \operatorname{Prin}\left(X, \Delta^{\mathrm{op}}\right) \simeq \operatorname{Lin}(X) \times \operatorname{Lin}(X) .
$$

Now, an augmentation is the same thing as a bisimplicial map from the product of two linear orders $N L \times N L^{\prime}$ to a bisimplicial set $Y$. Hence, similarly to Definition 2.7, we do have:

Definition 5.20. For any space $X$ and any bisimplicial space $Y$, write $\operatorname{Lin}^{2}(X, Y)$ for the product category of linear orders $\left(L \times L^{\prime}\right.$, aug) over $X$ equipped with a bisimplicial map (augmentation) aug: $N L \times N L^{\prime} \rightarrow Y$ from the product of nerves of $L$ and $L^{\prime}$ to $Y$. The morphisms $\left(L \times L^{\prime}\right.$, aug $) \rightarrow\left(L_{1} \times L_{1}^{\prime}, \operatorname{aug}^{\prime}\right)$ in $\operatorname{Lin}^{2}(X, Y)$ are maps of products of the linear orders $L \times L^{\prime} \rightarrow L_{1} \times L_{1}^{\prime}$ such that the induced map $N L \times N L^{\prime} \rightarrow N L_{1} \times N L_{1}^{\prime}$ on the products of nerves respects the augmentations.

With the same definition of concordance as in Definition 2.9, we do have similarly to Theorem 2.10:

Theorem 5.21. Let $Y$ be a bisimplicial space. For any space $X$ there is a natural equivalence of categories

$$
\operatorname{Hom}(\operatorname{Sh}(X), \operatorname{Sh}(Y)) \simeq \operatorname{Lin}^{2}(X, Y) .
$$

On homotopy classes of topos morphisms we have the natural bijection

$$
[\operatorname{Sh}(X), \operatorname{Sh}(Y)] \cong \operatorname{Lin}_{c}^{2}(X, Y) .
$$

Similarly to Theorem 2.3, we have the following theorem, where the geometric realization $|Y|$ of a a bisimplicial space $Y$ can be taken as the geometric realization of its diagonal. Equivalently, $Y$ can be defined as the "horizontal" geometric realization followed by the the "vertical" one or vice-versa. 
Theorem 5.22. For any bisimplicial space $Y$ the topoi $\operatorname{Sh}(Y)$ and $\operatorname{Sh}(|Y|)$ have the same weak homotopy type.

We recall that, in accordance with Remark 5.15, a bisimplicial space $Y$ is locally contractible if all spaces $Y_{n, m}$ are locally contractible. Again, geometric realization of a locally contractible bisimplicial space is locally contractible. Hence, we have similarly to 2.11 the following corollary:

Corollary 5.23. Let $X$ be a $\mathrm{CW}$-complex and $Y$ a locally contractible bisimplicial space. There is a natural bijection between homotopy classes of the maps $[X,|Y|]$ and the concordance classes $\operatorname{Lin}_{c}^{2}(X, Y)$.

\section{Classifying spaces and classifying topoi of topological bicat- egories II}

It is beyond the scope of this paper to give a full account of the constructions of Lack and Paoli, Tamsamani and Simpson. Concerning the latter two, the interested reader may find the nice survey of definitions of $n$-categories by T. Leinster [19]. Let Set and Cat ${ }_{1}$ denote the categories of (small) sets and (small) categories, respectively, and let Cat denote the 2-category of (small) categories.

\subsection{Lack-Paoli nerve as a singular functor}

The nerve construction of Lack and Paoli $[\mathbf{1 7}]$ is obtained as the singular functor when $\mathcal{V}=$ Cat. In order to define the nerve $N \mathbb{B}$ of a (small) bicategory $\mathbb{B}$, they introduced a (strict) 2-category NHom with bicategories as objects, whose 1-cells are normal homomorphisms (normal lax functors with invertible comparison maps). We will not give the general definition of 2-cells (icons) here. We describe them below explicitly in a special case.

Every category (in particular Cat $_{1}$ and the category defined by the ordinal $[n]$ ) can be seen as a locally discrete bicategory with only identity 2-cells. The normal homomorphism between locally discrete bicategories is just a functor between the corresponding categories, and there are no nontrivial icons between such functors. In this way, we obtain a fully faithful inclusion 2 -functor

$$
J: \Delta \rightarrow \text { NHom }
$$

and the category $\Delta$ can be seen as a full sub-2-category of NHom. The singular 2 -functor

$$
N_{L P}: \text { NHom } \rightarrow\left[\Delta^{\mathrm{op}}, \mathrm{Cat}\right]
$$

of the inclusion $J$ is a Lack and Paoli 2-nerve. The 2-functor $N_{L P}$ is fully faithful.

Definition 6.1. A normal homomorphism $(B, f, \beta):[n] \rightarrow \mathbb{B}$ from an ordinal $[n]$ to the bicategory $\mathbb{B}$ is a lax normal functor for which each 2 -cell $\beta_{i j k}$ in Definition 4.1 is invertible.

Definition 6.2. An icon between normal homomorphisms $F, G:[n] \rightarrow \mathbb{B}$ of bicategories is a lax natural transformation $\phi: F \Rightarrow G$, in which the component $\phi_{i}: B_{i} \rightarrow C_{i}$ is an identity, for each $i \in[n]$. More explicitly, an icon $\phi:(B, f, \beta) \Rightarrow(C, g, \gamma)$ consists of the following data: 
(i) For any $i \in[n]$ an identity $B_{i}=C_{i}$,

(ii) For each $i, j \in[n]$ such that $i \leqslant j$, a 2 -cell $\phi_{i j}: f_{i j} \Rightarrow g_{i j}$

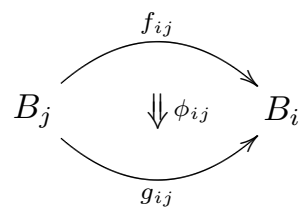

such that for all $i, j, k \in[n]$ with $i \leqslant j \leqslant k$ we have an equality of pasting diagrams
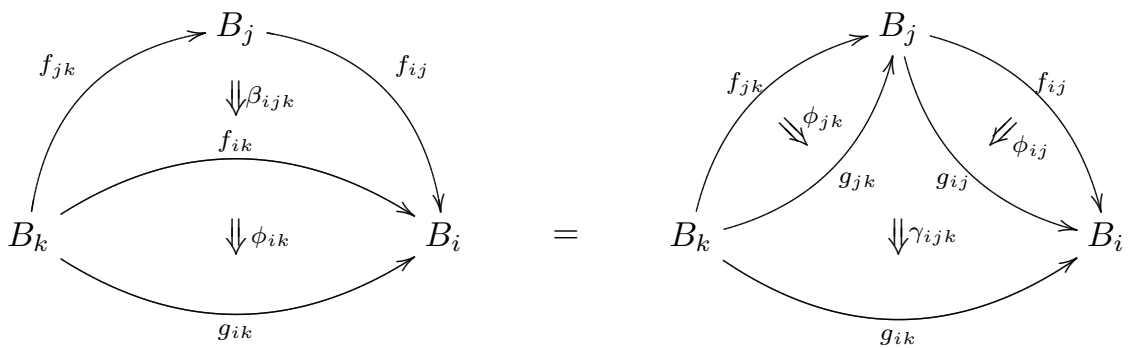

which means that the following identity of 2-cells holds in $\mathbb{B}$ :

$$
\phi_{i k} \beta_{i j k}=\gamma_{i j k}\left(\phi_{i j} \circ \phi_{j k}\right) \text {. }
$$

\subsection{Characterization of Lack-Paoli 2-nerves of bicategories}

In their paper [17], Lack and Paoli also described necessary and sufficient conditions for a simplicial object $X: \Delta^{\mathrm{op}} \rightarrow$ Cat to be a 2-nerve of a bicategory. In order to provide such characterization, they used discrete isofibrations which are functors $P: E \rightarrow B$ such that for each object $e$ in the category $E$ and each isomorphism $\beta: b \rightarrow P(e)$ in $B$ there exists a unique isomorphism $\varepsilon: e^{\prime} \rightarrow e$ in $E$ with $P(\varepsilon)=\beta$. Further, let $\mathrm{c}_{n}: X_{n} \rightarrow \operatorname{Cosk}_{n-1}(X)_{n}$ denote the $n$-component of the simplicial map $c: X \rightarrow \operatorname{Cosk}_{n-1}(X)$ from a simplicial object $X$ to its $n-1$-coskeleton $\operatorname{Cosk}_{n-1}(X)$, which is the unit of an adjunction between $(n-1)$-truncation $\operatorname{tr}_{n}$ and $(n-1)$-coskeleton $\operatorname{Cosk}_{n-1}$.

Theorem 6.3. Necessary and sufficient conditions for a 2-functor $X: \Delta^{\mathrm{op}} \rightarrow$ Cat to be a 2-nerve of a bicategory are:

(i) $X$ is 3-coskeletal,

(ii) $X_{0}$ is discrete,

(iii) the Segal functors $S_{n}: X_{n} \rightarrow X_{1} \times_{X_{0}} \cdots \times_{X_{0}} X_{1}$ are equivalences of categories,

(iv) $\mathrm{c}_{2}$ and $\mathrm{c}_{3}$ are discrete isofibrations. 


\subsection{Lack-Paoli 2-nerve as a bisimplicial set (space)}

If we apply the Grothendieck nerve functor at each level of the 2-nerve of Lack and Paoli (6.1), we obtain a functor

$$
B_{L P}: \mathrm{NHom} \rightarrow\left[\Delta^{\mathrm{op}}, \mathrm{SSet}\right],
$$

where the right-hand side is the category of bisimplicial sets. If we define the 2nerve in such bisimplicial terms, then the definition also makes sense for a topological bicategory $\mathbb{B}$, in which case the 2-nerve will naturally be a bisimplicial space. Although the above conditions 6.2 can be translated into the bisimplicial language, we will not do it here. From now on we will understand the 2-nerve of Lack and Paoli as a bisimplicial set (bisimplicial space in case of a topological bicategory).

\subsection{Tamsamani and Simpson}

Let Tam denote the full sub-2-category of [ $\Delta^{\mathrm{op}}$, Cat] consisting of those $X$, for which $X_{0}$ is discrete and the Segal maps $S_{n}$ are equivalences. Further, let Simpson denote the smaller full sub-2-category of those $X$ for which the Segal maps $S_{n}$ are fully faithful and surjective on objects. Also, in these cases we can interpret these "2-nerves" as bisimplicial sets (see $[\mathbf{1 9}, \mathbf{2 3}, \mathbf{2 5}]$, where the corresponding definitions can be found). We will speak of a Tamsamani 2-nerve (or 2-category) and a Simpson 2-nerve (or 2-category).

Remark 6.4. The Lack-Paoli 2-nerve is also a Simpson 2-nerve and thus also a Tamsamani 2-nerve. To each Tamsamani 2-nerve $X$ there is a bicategory $G X$ (and viceversa) constructed in [25]. For more details on Tamsamani 2-nerves (including a proper notion of equivalence), we refer the reader to this paper.

Here we only mention the following results of Lack and Paoli:

The (Lack-Paoli) 2-nerve 2-functor $N_{L P}: \mathrm{NHom} \rightarrow$ Tam, seen as landing in the 2-category Tam, has a left 2-adjoint $G$. Since $N_{L P}$ is fully faithful, the counit $G N_{L P} \rightarrow$ 1 is invertible. Each component $u: X \rightarrow N_{L P} G$ of the unit is a pointwise equivalence (i.e., each component $u_{n}$ is an equivalence) and $u_{0}$ and $u_{1}$ are identities.

Let $\operatorname{Ps}\left(\Delta^{\mathrm{op}}\right.$, Cat $)$ denote the 2-category of 2-functors, pseudonatural transformations and modifications, and let $\mathrm{Tam}_{\mathrm{ps}}$ be its full sub-2-category consisting of Tamsamani 2-categories. Then the 2-nerve 2-functor $N_{L P}: \mathrm{NHom} \rightarrow \mathrm{Tam}_{\mathrm{ps}}$ is a biequivalence of 2-categories.

Definition 6.5. Let $\mathbb{B}$ be a topological bicategory. The classifying topos $\mathcal{B}_{L P} \mathbb{B}$ of the topological bicategory $\mathbb{B}$ is defined as the topos of sheaves $\operatorname{Sh}\left(B_{L P} \mathbb{B}\right)$ on the bisimplicial space $B_{L P} \mathbb{B}$ (Lack-Paoli bisimplicial nerve).

Definition 6.6. The classifying space $B_{L P} \mathbb{B}$ of a topological bicategory $\mathbb{B}$ is the geometric realization $\left|B_{L P} \mathbb{B}\right|$ of its bisimplicial nerve $B_{L P} \mathbb{B}$.

With these definitions we have the following corollary of Theorem 5.22.

Corollary 6.7. For any topological bicategory $\mathbb{B}$, the topos of sheaves $\operatorname{Sh}\left(B_{L P} \mathbb{B}\right)$ on the classifying space $B_{L P} \mathbb{B}$ has the same weak homotopy type as the classifying topos $\mathcal{B B B}$. 
Definition 6.8. For a topological bicategory $\mathbb{B}$, write $\operatorname{Lin}^{2}(X, \mathbb{B})$ for the product category of linear orders over $X$ equipped with an augmentation aug: $N L \times N L^{\prime} \rightarrow$ $N N \mathbb{B}$. An object $E$ of this category will be called a Lack-Paoli principal $\mathbb{B}$-bundle. We call the two Lack-Paoli principal $\mathbb{B}$-bundles $E_{0}$ and $E_{1}$ on $X$ concordant, if there exists a Lack-Paoli principal $\mathbb{B}$-bundle on $X \times[0,1]$ such that we have the equivalences $E_{0} \simeq i_{0}^{*}(E)$ and $E_{0} \simeq i_{1}^{*}(E)$ under the obvious inclusions $i_{0}, i_{1}: X \hookrightarrow X \times[0,1]$.

Similarly to Theorems 3.6 and 4.9 we have from Theorem 5.21 the following "classifying" property of the classifying topos $\mathcal{B}_{L P} \mathbb{B}$.

Theorem 6.9. For a topological bicategory $\mathbb{B}$ and a topological space $X$, there is a natural equivalence of categories

$$
\operatorname{Hom}\left(\operatorname{Sh}(X), \mathcal{B}_{L P} \mathbb{B}\right) \simeq \operatorname{Lin}^{2}(X, \mathbb{B}) .
$$

On homotopy classes of topos morphisms we have the natural bijection

$$
\left[\operatorname{Sh}(X), \mathcal{B}_{L P} \mathbb{B}\right] \cong \operatorname{Lin}_{c}^{2}(X, \mathbb{B}) .
$$

The "classification" property of the classifying space $B_{L P} \mathbb{B}$ now follows as a corollary from Corollary 5.23.

Corollary 6.10. For a locally contractible bicategory $\mathbb{B}$ and a $\mathrm{CW}$-complex $X$, there is a natural bijection

$$
\left[X, B_{L P} \mathbb{B}\right] \cong \operatorname{Lin}_{c}^{2}(X, \mathbb{B}) .
$$

Remark 6.11 (Tamsamani and Simpson principal $\mathbb{B}$-bundles). In the above Definitions 6.5, 6.6 and 6.8 we could have used, instead of a Lack-Paoli 2-nerve, the Tamsamani or the Simpson 2-nerve (in the case these are bisimplicial spaces). Obviously, for such Tamsamani and Simpson principal BB-bundles, Corollaries 6.7, 6.10 and Theorem 6.9 are still valid.

\section{References}

[1] N.A. Baas, M. Bökstedt and T.A. Kro, Two-categorical bundles and their classifying spaces, arXiv:math/0612549.

[2] J.C. Baez and D. Stevenson, The classifying space of a topological 2-group, in Algebraic topology: The Abel symposium 2007 (N. Baas, E. Friedlander, B. Jahren and P. Arne Østvær, eds.), Springer-Verlag, New York, 2009.

[3] I. Baković, Bigroupoid 2-torsors, Ph.D. thesis, Ludwig-Maximilians-Universität Munich, 2008.

[4] I. Baković, The simplicial interpretation of bigroupoid 2-torsors, arXiv: 0902.3436.

[5] I. Baković and B. Jurčo, The Diaconescu's theorem for 2-topoi, in preparation.

[6] J. Bénabou, Introduction to bicategories, 1967 reports of the Midwest category seminar (Bénabou et al., eds.), Lecture Notes in Math. 47, Springer-Verlag, New York, 1967.

[7] R. Brown and P.J. Higgins, The equivalence of $\infty$-groupoids and crossed complexes, Cah. Top. Géom. Diff. 22 (1981), no. 4, 371-386. 
[8] R. Brown and P.J. Higgins, The classifying space of a crossed complex, Math. Proc. Camb. Phil. Soc. 110 (1991), no. 1, 95-120.

[9] R. Brown, M. Golasinski, T. Porter and A. Tonks, Spaces of maps into classifying spaces for equivariant crossed complexes, Indag. Math. (N.S.) 8 (1997), no. 2, 157-172.

[10] R. Brown, M. Golasinski, T. Porter and A. Tonks, Spaces of maps into classifying spaces for equivariant crossed complexes. II. The general topological group case, K-Theory 23 (2001), no. 2, 129-155.

[11] J. Duskin, Simplicial matrices and the nerves of weak $n$-categories. I. Nerves of bicategories, Theory and applications of categories 9 (2000), 198-308.

[12] N. Gurski, Nerves of bicategories as stratified simplicial sets, J. Pure Appl. Algebra 213 (2009), no. 6, 927-946

[13] J.R. Isbell, Adequate subcategories, Illinois J. Math. 4 (1960), 541-552.

[14] J.R. Isbell, Subobjects, adequacy, completeness and categories of algebras, Rozprawy Mat. 36 (1964), 3-33.

[15] B. Jurčo, Crossed module bundle gerbes; classification, string group and differential geometry, arXiv:math/0510078.

[16] G.M. Kelly, Basic concepts of enriched category theory, London Math. Soc. Lecture Note Series 64, Cambridge University Press, New York, 1982, 245 pp.

[17] S. Lack and S. Paoli, 2-nerves for bicategories, K-Theory 38 (2008), no. 2, 153-175.

[18] J. Lurie, Higher topos theory, Ann. of Math. Studies 170, Princeton University Press, Princeton, NJ, 2009.

[19] T. Leinster, A survey of definitions of n-category, Theory Appl. Categ. 10 (2002), 1-70.

[20] S. Mac Lane and I. Moerdijk, Sheaves in geometry and logic. A first introduction to topos theory, Universitext, Springer-Verlag New York, 1992.

[21] I. Moerdijk, Classifying spaces and classifying topoi, Lecture Notes in Math. 1616, Springer-Verlag, New York, 1995.

[22] G. Segal, Classifying spaces related to foliations, Topology 17 (1978), 367-382.

[23] C. Simpson, A closed model structure for $n$-categories, internal hom, $n$-stacks and generalized Seifert-Van Kampen, alg-geom/9704006, 1997.

[24] R. Street, The algebra of oriented simplexes, J. Pure Appl. Algebra 49 (1987), no. $3,283-335$.

[25] Z. Tamsamani, Sur des notions de $n$-catégorie et $n$-groupoïde non strictes via des ensembles multi-simpliciaux, K-Theory 16 (1999), no. 1, 51-99.

[26] R.W. Thomason, Homotopy colimits in the category of small categories, Math. Proc. Camb. Phil. Soc. 85 (1979), no. 1, 91-109.

[27] D. Verity, Complicial sets characterising the simplicial nerves of strict $\omega$-categories, Memoirs Amer. Math. Soc. 193, A. M. S., Providence, RI, 2005.

[28] D. Verity, Weak complicial sets. I. Basic homotopy theory, Adv. Math. 219 (2008), no. 4, 1081-1149. 
[29] D. Verity, Weak complicial sets. II. Nerves of complicial Gray-categories, in Categories in algebra, geometry and mathematical physics, 441-467, Contemp. Math. 431, A. M. S., Providence, RI, 2007.

[30] M. Weiss, What does the classifying space of a category classify?, Homology Homotopy Appl. 7 (2005), no. 1, 185-195.

Igor Baković ibakovic@gmail.com

Faculty of Natural Sciences and Mathematics, University of Split, Teslina 12/III, 21000 Split, Croatia

Branislav Jurčo branislav.jurco@googlemail.com

Max Planck Institute for Mathematics, Vivatsgasse 7, 53111 Bonn, Germany 